\title{
AUTOMUTILAÇÃO DEVIDO À COMPRESSÃO DA CAUDA EQÜINA EM TRÊSS CÃES E UM GATO
}

\section{SELF-MUTILATION IN THREE DOGS AND ONE CAT WITH CAUDA EQUINA COMPRESSION}

\author{
Cynthia Mary Gomes Lagedo ${ }^{1}$ Eduardo Alberto Tudury ${ }^{2}$ \\ Maria de Lourdes Estrela Faria ${ }^{3}$
}

\section{RESUMO}

O presente trabalho relata a ocorrência de dermatite prurítica acral e automutilação da cauda e região genitocrural em três cães e um gato que sofriam compressão de raízes nervosas da cauda eqüina. $O$ diagnóstico foi realizado através dos sinais clínicos e por meio de exames radiográficos. O tratamento consistiu na descompressão cirúrgica da cauda eqüina, através de laminectomia dorsal, conseguindo-se com A mesmA, a remissão total das automutilações e perseguições da cauda, com cicatrização das lesões auto-infligidas.

Palavras-chaves: doenças da coluna vertebral, síndrome da cauda eqüina, automutilação.

\section{SUMMARY}

This paper describes the occurrence of acral pruritic dermatitis and self-mutilation of the tail, hindlimbs and genitalia areas in three dogs and one cat with compression of the cauda equina nerve roots. The diagnostic was carried out by clinical signs and radiological studies. The treatment was based upon the surgical decompression of the cauda equina by the dorsal laminectomy. The surgical treatment led to the total remission of the self-mutilation, tail persecution and the self-inflicted lesions.

Key words: spinal diseases, cauda equina syndrome, selfmutilation.

\section{INTRODUÇÃO}

Automutilação (auto-agressão), caracterizada por lambedura, mastigação, arranhadura e fricção de uma parte do corpo, pode ser considerada um distúrbio comportamental (PARKER, 1992). A lambedura excessiva pode causar a produção e liberação de endorfinas, fazendo o animal sentir-se melhor (eufórico) e ao mesmo tempo produzindo um efeito analgésico que diminui a percepção de dor. Estes efeitos levam os animais a se lamber compulsivamente (SCOTT et al., 1996). A síndrome de automutilação pode ser uma das desordens mais terríveis de serem suportadas pelos proprietários de animais, e das mais desafiadoras e frustrantes para o veterinário diagnosticar e tratar (CHRISMAN, 1991).

As causas de automutilação podem ser orgânicas e não orgânicas. Dentre elas, têm-se as dermatopatias (SCOTT et al., 1996); psicogênicas e neuropatias (PARKER, 1992); epilepsias sensoriais, mielopatias e ganglioneuropatias (CHRISMAN, 1991), assim como a síndrome da cauda eqüina (KOMAREK, 1988; BRAUND, 1994).

A síndrome da cauda equiina é expressão que engloba um amplo espectro de distúrbios, congênitos ou adquiridos, provocadores de estreita-

\footnotetext{
${ }^{1}$ Médico Veterinário, Mestranda do Departamento de Medicina Veterinária, Universidade Federal Rural de Pernambuco, Área de Clínica.

${ }^{2}$ Médico Veterinário, Doutor, Professor Adjunto I, Departamento de Medicina Veterinária, Universidade Federal Rural de Pernambuco, Rua Dom Manoel de Medeiros, s/nº, Bairro Dois Irmãos, 52171-900 Recife, PE. E-mail: tudury@ nelore.npde.ufrpe.br. Autor para correspondência.

${ }_{3}^{3}$ Médico Veterinário, PhD., Professor Adjunto IV, Departamento de Clínicas Veterinárias, Universidade Estadual de Londrina, Londrina-PR.
} 
mento do canal vertebral lombossacro, com resultante compressão das raízes nervosas da cauda eqüina (SCHULMAN \& LIPPINCOTT, 1988; LECOUTER \& CHILD, 1992). Os distúrbios congênitos são conformados por anomalias vertebrais ou das raízes nervosas. Como distúrbios adquiridos foram citados estenoses do canal vertebral lombossacro degenerativas e pós-cirúrgicas, comprometimentos vasculares, instabilidade, subluxações, patologias do disco intervertebral, discoespondilites, estenoses traumáticas (fraturas e luxações) e neoplasias (INDRIERI, 1988; SCHULMAN \& LIPPINCOTT, 1988; LENEHAM \& TARVIN, 1990; PALMER \& CHAMBERS, 1991; PRATA, 1993; FOSSUM, 1997). Por vezes, podem ocorrer combinações de anomalias congênitas e adquiridas, estenosando o canal vertebral lombossacro (LECOUTER \& CHILD, 1992).

São sintomas de compressão da cauda eqüina em cães e gatos: dor à palpação e extensão da articulação lombossacra, paraparesias, atrofias de músculos dos membros pélvicos, claudicação freqüentemente unilateral destes membros, paresia da cauda, perdas de propriocepção nos membros posteriores, incontinências urinária e/ou fecal assim como automutilações do períneo, cauda (junto com perseguição da mesma), genitália e membros pélvicos (KOMAREK, 1988; SCHULMAN \& LIPPINCOTT, 1988; LENEHAM \& TARVIN, 1990; PRATA, 1993; BRAUND, 1994).

As parestesias (distúrbios sensoriais desprazerosos causadores de automutilação) existentes na estenose do canal vertebral lombossacro podem decorrer da irritação de fibras sensoriais da cauda eqüina provenientes de dermátomos inervados pelos nervos pudendo, ciático e caudais (INDRIERI, 1988; MORGAN \& BAILEY, 1990; PRATA, 1993). Outros distúrbios que desencadeiam automutilação da cauda, genitália e membros pélvicos são: dermatopatias, epilepsias, neuroma de caudectomia, orquites, ganglioradiculoneuropatias e neuropatias sensoriais, assim como a síndrome de hiperestesia idiopática (GREENE, 1984; CHRISMAN, 1991; SCOTT et al., 1996). Em geral, cães com lesões decorrentes da parestesia, gerada pela compressão da cauda eqüina, são primeiramente tratados contra problemas dermatológicos mal definidos (LENEHAM \& TARVIN, 1990).

O diagnóstico da síndrome da cauda equiina baseia-se nos sinais clínicos característicos, nos resultados dos exames radiográficos simples e contrastados e, por vezes, nos achados da laminectomia exploratória (INDRIERI, 1988; LECOUTER \& CHILD, 1992; BRAUND, 1994). Nas radiografias podem ser identificados: espondilose deformante ventral e lateral à articulação lombossacra, esclerose das placas vertebrais terminais, "encunhamento" ou estreitamento do disco intervertebral lombossacro, osteoartrose das facetas articulares, deslocamento ventral do sacro com respeito a $L_{7}$, dimensões dorso-ventrais diminuídas do canal vertebral lombossacro, assim como sinais radiográficos, caracterizando discoespondilite, malformações vertebrais, fraturas, neoplasias, protrusão do disco intervertebral, etc. (SCHULMAN \& LIPPINCOTT, 1988; MORGAN \& BAILEY, 1990; LECOUTER \& CHILD, 1992; PRATA, 1993). Estudos radiográficos contrastados, como epidurografia, mielografia, discografia, venografia vertebral e tomografia computadorizada, também têm sido recomendados, para o diagnóstico de compressões da cauda eqüina, já que as radiografias simples podem levar a diagnósticos falsos negativos e falsos positivos (MORGAN \& BAILEY, 1990; LECOUTER \& CHILD, 1992; PRATA, 1993).

$\mathrm{O}$ tratamento mais preconizado para aliviar os sintomas decorrentes de compressão das raízes da cauda eqüina é a laminectomia dorsal lombossacra, acompanhada, quando necessário, de facetectomia, foraminotomia, fenestração dorsal de discos intervertebrais e/ou estabilização vertebral (LECOUTER \& CHILD, 1992; PRATA, 1993, FOSSUM, 1997). KOMAREK (1988), através de laminectomia dorsal lombossacra, debelou um quadro de automutilação e perseguição da cauda que acometia um cão, Pastor alemão, de 19 meses de idade. Por vezes, nestes casos, torna-se necessária a realização de caudectomia, em decorrência das graves lesões já infligidas (PRATA, 1993).

O presente trabalho tem como objetivo relatar quatro casos de síndrome da cauda eqüina, onde os animais, concomitantemente, exibiam parestesias automutilantes, que foram cirurgicamente solucionadas.

\section{RELATO DOS CASOS}

Para todos os pacientes foi preenchida uma ficha, contendo informações de resenha, anamnese e sinais clínico-neurológicos, na qual foram também transcritos os resultados dos exames complementares e dos tratamentos efetuados. A seguir, são individualmente apresentadas estas informações, para melhor compreensão dos casos:

CASO 1: tratou-se de um canino, macho, sem raça definida (srd), 5 anos de idade e $10 \mathrm{~kg}$ de peso, que apresentava agressividade exacerbada para com os proprietários e perseguição da cauda. Ao exame clínico foi constatado prurido intenso, automutilação e feridas ulceradas da mesma, assim como ausência 
de outras anormalidades clínico-neurológicas. Radiograficamente foi encontrada uma sacralização da coccígea $1\left(\mathrm{Co}_{1}\right)$ com angulação dorsal e estenose do canal vertebral em sacral $3\left(\mathrm{~S}_{3}\right)-\mathrm{Co}_{1}$ e $\mathrm{Co}_{1}-\mathrm{Co}_{2} . \mathrm{Na}$ epidurografia, o contraste não ultrapassou o local de estenose, extravasando-se epiaxialmente em $\mathrm{S}_{3}-\mathrm{Co}_{1}$.

CASO 2: tratou-se de um canino, macho, SRD, 3 anos de idade e $8 \mathrm{~kg}$ de peso, apresentando dores à extensão da articulação lombossacra e à compressão ventral do processo espinhoso da vértebra lombar 7 $\left(\mathrm{L}_{7}\right)$, porém com respostas normais ao exame neurológico. Além disto, exibia granuloma prurítico acral por lambedura no membro posterior esquerdo, o qual já havia sido tratado anteriormente e com insucesso, com vários protocolos para doenças cutâneas. Apresentava radiograficamente ( $\mathrm{Rx}$ simples) uma sacralização da $\mathrm{L}_{7}$, colapso, osteofitose e hérnia dorsal no disco intervertebral $\mathrm{L}_{6}-\mathrm{L}_{7}$, junto com hérnia discal dorsal em $\mathrm{L}_{5}-\mathrm{L}_{6}$.

CASO 3: tratou-se de um canino, macho, SRD, 2 anos e 6 meses de idade, $18 \mathrm{~kg}$ de peso, sobre o qual o proprietário relatava automutilação da cauda, um ano após o animal ter fugido para a rua e retornado apresentando dor e cifose lombossacra. Ao exame clínico-neurológico, só foram constatadas perseguição e mutilação da cauda, lesões por lambedura no escroto, junto com ulcerações, infecção e necrose da extremidade da cauda. Nas radiografias simples, o paciente apresentou fratura crônica em nível de $S_{2^{-}}$ $\mathrm{S}_{3}$, com estenose do canal vertebral nesse local, assim como esclerose das placas vertebrais terminais e osteofitoses ventrais em $\mathrm{L}_{7}-\mathrm{S}_{1}$.

CASO 4: tratou-se de um felino, macho, SRD, 3 anos e 6 meses de idade, $2 \mathrm{~kg}$ de peso, o qual, apesar de lamber constantemente a genitália, ânus, e face medial dos membros posteriores, não apresentava outras anormalidades clínico-neurológicas, fora da dor à extensão e à palpação dorsais da articulação lombossacra. Ao raio X simples, exibia uma subluxação ventral (provavelmente traumática) das vértebras coccígeas com relação ao sacro.

Caudectomia terapêutica foi realizada somente no caso de número 3 . Em todos os pacientes, efetuou-se uma abordagem dorsal à coluna vertebral, e mediante o uso de pinça goiva do tipo Stille Luer, realizou-se laminectomia dorsal descompressiva, do canal vertebral lombossacro, a qual foi estendida até as vértebras coccígeas, nos casos de número 4 e 2 . Neste último cão, realizou-se também fenestração dorsal dos discos intervertebrais L5-L6 e L6-L7, utilizando-se para isto lâmina de bisturi de número 11 e removedor de tártaro, enquanto, com reparos, mantinham-se afastadas as raízes da cauda equiina. O regime anestésico utilizado, em todos os casos, consistiu em pré-medicação com sulfato de atropina $(0,05 \mathrm{mg} / \mathrm{kg})$, seguida, 15 minutos após, da administração de cloridrato de xilazina $(1 \mathrm{mg} / \mathrm{kg})$, ambos pela via subcutânea, seguidos de thiopental sódico (aproximadamente 12,5mg/kg), pela via intravenosa, até o terceiro estágio de Guedel.

\section{RESULTADOS E DISCUSSÃO}

A suspeita de que compressão de raízes nervosas da cauda eqüina estava gerando a automutilação deste pacientes se baseou no fato que os sintomas de dor à palpação e extensão da articulação lombossacra e/ou parestesias na cauda, genitália e membros pélvicos já tinham sido descritos como característicos de síndrome da cauda eqüina por KOMAREK (1988), SCHULMAN \& LIPPINCOTT (1988), LENEHAM \& TARVIN (1990), PRATA (1993) e BRAUND (1994). O diagnóstico foi primeiramente concluído, conforme sugerido por INDRIERI (1988), LECOUTER \& CHILD (1992), BRAUND (1994), através de exames radiográficos, onde foram achados sinais típicos desta doença (SCHULMAN \& LIPPINCOTT, 1988; MORGAN \& BAILEY, 1990; LECOUTER \& CHILD, 1992; PRATA, 1993) como malformações vertebrais, estenoses do canal vertebral, osteofitoses e escleroses de placas vertebrais terminais, hérnias de disco, fraturas e difusão interrompida do contraste na epidurografia.

A laminectomia dorsal lombossacra descompressiva realizada em todos estes animais, junto com a fenestração do disco realizada no caso 2, indicadas por KOMAREK (1988), LECOUTER \& CHILD (1992); PRATA (1993) e FOSSUM (1997) para pacientes com síndrome da cauda eqüina, permitiu debelar os distúrbios sensoriais causadores de automutilação, levando à cicatrização total das lesões auto-infligidas. Além disto, no procedimento cirúrgico, frente às lesões estenosantes encontradas no transcorrer das laminectomias, e concordando com o exposto por INDRIERI (1988); LECOUTER \& CHILD (1992), confirmou-se o diagnóstico de compressão de raízes da cauda eqüina. A gravidade das lesões, encontradas na cauda do caso de número 3, obrigou a realização de caudectomia terapêutica, preconizada por PRATA (1993) nestes quadros, como complementar à laminectomia.

Realmente, a síndrome de automutilação em cães e gatos, conforme citado por CHRISMAN (1991), é muito desafiadora e frustrante para os Médicos Veterinários diagnosticar e tratar. Com relação àquela gerada pela compressão da cauda 
eqüina, verificou-se na anamnese do caso de número 2, estar certa a afirmação feita por LENEHAM \& TARVIN (1990), de que comumente estas parestesias são primeira e erroneamente tratadas como dermatopatias, esquecendo-se de que a irritação das raízes dos nervos ciáticos, pudendos e caudais podem levar à automutilação dos membros pélvicos; genitália, ânus e períneo, ou cauda, respectivamente (INDRIERI, 1988; MORGAN \& BAILEY, 1990; PRATA, 1993). Por tanto, e como conclusão desta experiência clínica, cabe ressaltar que, ao atender cães e gatos sofredores de perseguição da cauda, parestesias e automutilações de dermátomos correspondentes a raízes nervosas da cauda eqüina, devese pensar em compressões desta estrutura nervosa, junto às outras patologias (já citadas na revisão da literatura) geradoras destes sintomas.

\section{REFERÊNCIAS BIBLIOGRÁFICAS}

BRAUND, K.G. Clinical syndromes in veterinary neurology. 2. ed. St. Louis: Mosby, 1994. 476 p.

CHRISMAN, C.L. Self-mutilation. In: CHRISMAN, C.L. Problems in small animal neurology. 2. ed. Philadelphia: Lea \& Febiger, 1991. cap. 20, p. 469-477.

FOSSUM, T.W. Surgery of the lumbosacral spine. In: FOSSUM, T.W. Small animal surgery. St. Louis: Mosby, 1997. Cap. 36, p. 1131-1149.

GREENE, C.E. Clinical microbiology and infectious disease of the dog and the cat. Philadelphia: Saunders, 1984, 967 p.
INDRIERI, R.J. Lumbosacral stenosis and injury of the cauda equina. Veterinary Clinics of North America: Small Animal Practice, v. 18, n. 3, p. 697-710, 1988.

KOMAREK, J.V. Fallbericht: Verfolgung der rute beim hundcauda-equina-syndrom. Klienterpraxis, v. 33 , n. 1 , p. 25 , 1988

LECOUTER, R.A., CHILD, G. Moléstias da medula espinal. In: ETTINGER, S.J. Tratado de medicina interna veterinária. 3. ed. Rio de Janeiro: Manole, 1992. Cap. 62, p. 655-736.

LENEHAM, T.M., TARVIN, G.B. Surgical treatment of cauda equina compression syndrome. In: BOJRAB, M.J. Current techniques in small animal surgery. 3 . ed. Philadelphia: Lea \& Febiger, 1990. p. 629-635.

MORGAN, J.P., BAILEY, C.S. Cauda equina syndrome in the dog: Radiographic evaluation. Journal of Small Animal Practice, v. 31, n. 1, p. 69-76, 1990.

PALMER, R.H., CHAMBERS, J.N. Canine lumbosacral diseases Compendium on Continuing Education. Small Animal Practice, v. 13, n. 1, p. 61-69, 1991.

PARKER, A.J. Sintomas comportamentais de afecção orgânica In: ETTINGER, S.J.Tratado de medicina interna veterinária. 3. ed. Rio de Janeiro: Manole, 1992. Cap. 16, p. 74-78.

PRATA, R.G. Cauda equina syndrome. In: SLATTER, D. Textbook of small animal surgery. 2. ed. Philadelphia: Saunders, 1993. Cap. 77, p. 1094-1105.

SCHULMAN, A.J., LIPPINCOTT, C.L. Canine cauda equina syndrome. Compendium on Continuing Education. Small Animal Practice, v. 10, n. 7, p. 835-844, 1988.

SCOTT, D.W., MILLER, W.H., GRIFFIN, C.E. Muller \& Kirk. Dermatologia de pequenos animais. 5. ed. Rio de Janeiro: Interlivros, $1996.1130 \mathrm{p}$.

Ciência Rural, v. 29, n. 1, 1999. 